\title{
Novel and simple reversed lateral shear interferometer with single-cube beam splitter and spatial Fourier transform for symmetric lens measurement
}

\author{
Jianfei Sun ${ }^{1}$, Cheok Peng Lee ${ }^{2}$, Chee Khian $\mathrm{Ang}^{3}$, Weijuan $\mathrm{Qu}^{4}$ \\ \{LEE_Cheok_Peng@np.edu.sg $\left.{ }^{2}\right\}$ \\ School of Engineering, Ngee Ann Polytechnic, 535 Clementi Road, 599489 Singapore ${ }^{1234}$
}

\begin{abstract}
This paper presents a simple reversed lateral shearing interferometer system for symmetric lens measurement. Reversed lateral shearing are recorded by tilting a singlecube beam splitter, which introduced the spatial carrier frequency. Fourier method is applied to evaluate the phase of symmetric object, which is separated on the frequency domain after Fourier transform. This system can generate shearing graph by using a single image. The effects of shearing distance, the choice of algorithm and comparison result are discussed in detail. The theory and the application are presented.
\end{abstract}

Keywords: lateral shearing interferometer, quantitative, collimated beam, spatial phaseshift, phase curvature, numerical reconstruction, wavefront, first-order derivatives, symmetric axis, fringes, self-reference, temporal phase shifting, common path, digital shearogram.

\section{Introduction}

Lateral shearing interferometer with a microscope objective shows great success in the quantitative study of in diverse applications such as the testing of optical components and systems and the study of flow and diffusion phenomena in gases and liquids[1][2]. Basically, the method of lateral shearing interferometry consists of duplicating wavefront under study, displacing it laterally by a small amount, and obtaining the interference pattern between the original and the displaced wavefront.

There are several methods to get lateral shearing, and the most common one is using modified Jamin interferometer or Michelson Interferometer [3][4]. In the later years, using gratings of slightly different frequency where the overall grating frequency is high enough to separate the orders. Joenathan and Pedrini developed a holographic lenses based spatial phaseshift lateral shear system in 2013[5][6]. This system utilizes two holographic lenses in a collimated beam to get the shearing. However, in the case of the holographic lens, the shear is variable if the lenses are recorded on different plates. The fringes used as carrier for the wavefront information are always changing. In addition to that, with the applicant of microscope objective in Lateral shearing interferometer, it also introduces a phase curvature to the object wave that increases the complexity of the numerical reconstruction process. It would be hard to reconstruct the wavefront with variable distance of recorded plate. In 2009 Weijuan Qu and 
Anand Asundi developed a transparent digital holograms system, which is based on a single cube beam splitter (SCBS) interferometer[7][8]. The main advantage of this system is that it is common path. More precisely, the beam is geometrically split between a reference part of the sample and the area under investigation. Thus, by using a symmetry axis of the lens array and overlapping this two parts as a self-reference, we can build a reversed lateral shearing system for first-order derivatives of the wavefront investigation. This allows much simpler measurement of the symmetric objects.

In this paper, we report a relatively simple shearing interferometer that uses the SCBS tilt and allow for quantitative measurement at the same time. Modified SCBS interferometer provide an approach to manipulate the wavefront of light field. The tilt fringes are used as spatial frequency carrier, and therefore we use Fourier transform method to extract the phase information. This is unlike any other method of quantitative analysis where temporal phase shifting is required to extract the phase information. However, in this simple interferometer, the spatial frequency is used. The technique therefore lends itself to the measurement of transient changes. Consequently, the interferometer can be used for studying instantaneous changes or testing under harsh environment. Presented in this paper are the fundamentals behind the interferometer followed by experimental results.

\section{Experimental Setup and Theory}

In this paper we propose a lateral shearing interferometer based on a single cube beam splitter (SCBS) interferometer [9] using a microscope objective to provide magnification of the test specimen. An SCBS works in a nonconventional configuration so as to both split and combine a diverging spherical wavefront emerging from the microscope objective. As it is a common-path interferometer, the reference arm travels the same distance as the object arm. Their curvature is physically compensated by each other during interference. The unique features of the proposed setup are its simplicity, minimal number of optical elements, and insensitivity to vibration. The transmission mode of our setup are given as shown in Fig. 1. A high-resolution image is obtained by using an MO (an aspheric lens). The image formation is illustrated by using the dashed rays in the transmission mode setup. An image is obtained. The use of the beam splitter (BS) cube makes the whole optical setup a symmetrical common-path interferometer. Light incident on the front cube wall will change the propagation direction inside the BS. Only the refracted light, which arrives at the central semi-reflecting layer and is reflected to the back cube wall, can be output through the BS. Other rays will travel from the front cube wall directly to the back cube wall and will suffer from total reflection. Because of the small angle $\theta$ between the light propagation direction and the central semi-reflecting layer, a wedge shaped optical path difference will be introduced to the reflected light and the transmitted light. Thus in the interference plane, one can achieve interferogram with a straight fringe pattern. The fringe pattern is worked as a stable special carrier which will not varying with the position of the object. 


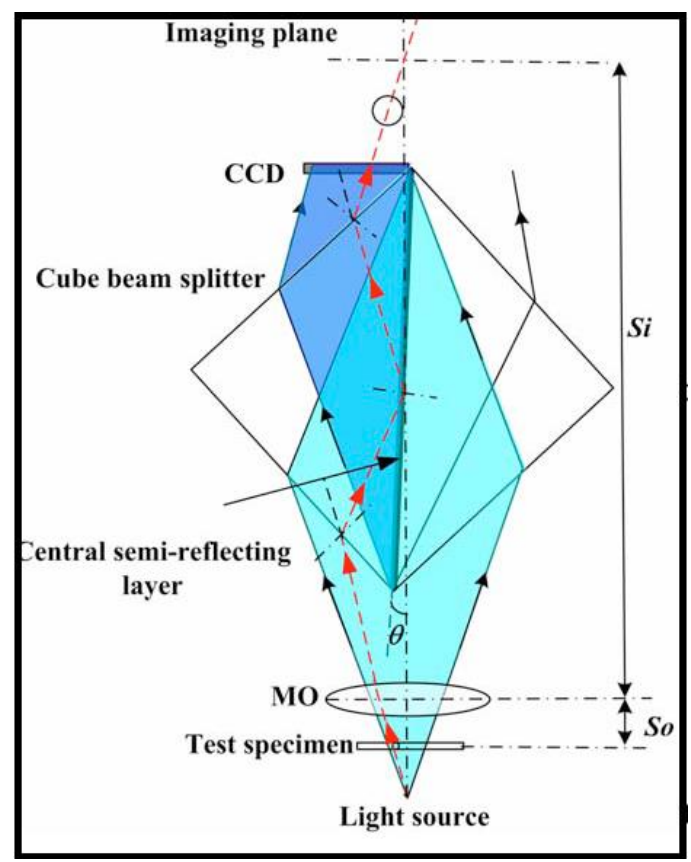

Fig. 1. Schematic of the proposed setup.

As it is a self-interference in lateral shearing interferometer, the reference wave is reversed in the image plane. Regarding all the lenses in the lens array are uniform, the reversed image can be ignored. When a symmetric reference wavefront is used for the reconstruction of the recorded digital shearogram, the first-order derivatives of the wavefront is achieved. Here a brief theoretical description is given for the case in which shearogram are captured by CCD image. The CCD camera captures two wave fronts from the reference beam and object beam. In this experiment, the reference beam is a spherical wave produced by a single-mode optical fiber. Consider the object wave $U_{1}(\xi, \eta)$ and its matching lateral shearing reference wave $U_{2}(\xi, \eta)$, where $\xi$ and $\eta$ are rectangular coordinates at the shearogram plane detected by the CCD sensor. The complex amplitude of these two wave fronts can be represented by the following equations.

$$
\begin{gathered}
U_{1}(\xi, \eta)=u_{1}(\xi, \eta) \exp \left[i \theta_{k}(\xi, \eta)\right] \\
U_{2}(\xi, \eta)=u_{2}(\xi+\Delta \xi, \eta+\Delta \eta) \exp \left[i \varphi(\xi+\Delta \xi, \quad \eta+\Delta \eta)-2 \pi i\left(f_{\xi} \xi+f_{\eta} \eta\right)\right]
\end{gathered}
$$

Where $f_{\xi}$ and $f_{\eta}$ are spatial carrier frequencies in the $\xi$ and the $\eta$ directions, resulting from the slightly tilted cube beam splitter. The total intensity recorded on each of the CCD detectors is an superposition of the object beam and the reference beam corresponding to each direction and is given by

$I(\xi, \eta)=U_{1}(\xi, \eta)+U_{2}(\xi+\Delta \xi, \quad \eta+\Delta \eta)^{2}=U_{1}(\xi, \eta)^{2}+U_{2}(\xi+\Delta \xi, \quad \eta+\Delta \eta)^{2}$ 


$$
+U_{1}(\xi, \eta) U_{2}(\xi+\Delta \xi, \eta+\Delta \eta)+U_{2}(\xi+\Delta \xi, \quad \eta+\Delta \eta) U_{1}(\xi, \eta)
$$

The * denotes the amplitude complex conjugate of each beams. By substituting Eq. (1) and Eq. (2) into Eq. (3) the total intensity can be written as

$$
\begin{aligned}
& I(\xi, \eta)=u_{1}^{2}(\xi, \eta)+u_{2}^{2}(\xi+\Delta \xi, \quad \eta+\Delta \eta)+c_{k}(\xi, \quad \eta) \exp \left[2 \pi i\left(f_{\xi} \xi+f_{\eta} \eta\right)\right]+ \\
& c_{k}(\xi, \eta) \exp \left[-2 \pi i\left(f_{\xi} \xi+f_{\eta} \eta\right)\right]
\end{aligned}
$$

Where

$$
c_{k}(\xi, \eta)=u_{1}(\xi, \eta) u_{2}(\xi+\Delta \xi, \eta+\Delta \eta) \exp [i \varphi(\xi, \eta)]
$$

The last but two terms of Eq. (4) describe the each of three sets of carrier interference fringes with spatial frequencies $\left(f_{1}, f_{2}\right)$ and modulated in phase by $\varphi(\xi, \eta)$. The FT converts the image from the spatial domain to the Fourier domain. Application of the two-dimensional FT to Eq. (4) transforms the equation into the following form.

$$
\begin{gathered}
F T\left[I_{k}(\xi, \eta)\right]=A_{k}\left(f_{1}, f_{2}\right)+C_{k}\left(f_{1}-f_{\xi}, f_{2}-f_{\eta}\right)+C_{k}\left(f_{1}+f_{\xi}, f_{2}+f_{\eta}\right) \\
A_{k}(\xi, \eta)=F T\left[u|\quad| 1^{2}(\xi, \eta)+u_{2}^{2}(\xi, \eta)\right]
\end{gathered}
$$

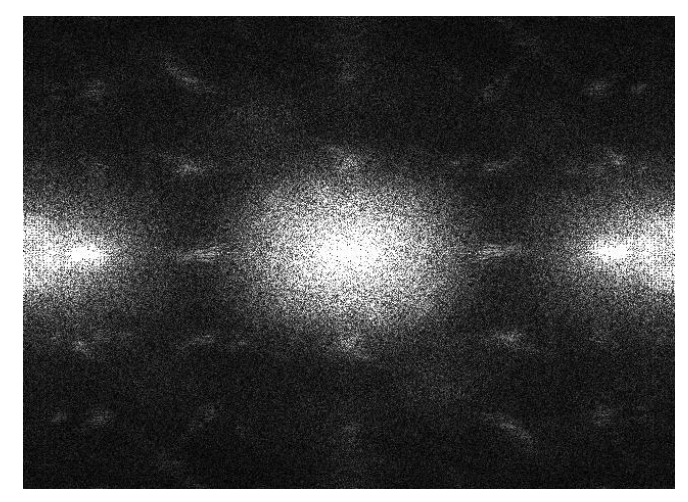

Fig. 2. Fourier spectrum of the shearogram.

Fig. 2 shows the Fourier spectrum of the images. There are three spectra on the Fourier domain, which can be clearly identified. From the spectrum, it can be seen that the four terms are separated into three parts due to different spatial frequencies. The term $A_{k}(\xi, \eta)$ which is low frequency terms mainly from the background light, is located at the center of the frequency domain. The terms $C_{k}$ and $C_{k}$ describe a complex conjugate pair, each containing the required phase information $\varphi(\xi, \eta)$ of the recorded hologram located at $\left(f_{\xi}, f_{\eta}\right)$ and $\left(-f_{\xi},-f_{\eta}\right)$. These terms are shifted in the Fourier domain and can, by appropriate choice of carrier frequencies. By applying a Inverse Fourier Transform on the circled area located at $\left(f_{\xi}, f_{\eta}\right)$, the phase distributions can be calculated using the complex amplitudes. This results in the following equation. 


$$
\varphi(\xi, \eta)-\varphi(\xi+\Delta \xi, \quad \eta+\Delta \eta)=\arctan \left\{\frac{\mathfrak{I}\left[c_{k}(\xi, \eta)\right]}{\Re\left[c_{k}(\xi, \eta)\right]}\right\}
$$

For Eq. (8), Im and Re denote the imaginary and real part of the complex numbers. Assuming the shearing distance to be very small and the shearing direction is along $\mathrm{X}$ direction. The relative phase due to shearing can then be calculated as the derivative of the testing objects.

$$
\varphi_{x}=\frac{4 \pi d x}{\lambda} \frac{\partial z}{\partial x}
$$

Where the $\varphi_{x}$ is the phase of wavefront $d x$ is the shear amount in the system $\lambda$ is the wavelength of the laser. By adjusting $d x$, different sensitivity of $\frac{\partial z}{\partial x}$ can be achieved, which representing the slope of the surface.

\section{Discussion and Application}

The main advantage of this system is that it is common path. More precisely, the beam is geometrically split between a reference part of the sample and the area under investigation. In this case, the shearing amount is totally depend on the location of the specimen. The key of the spatial phase shift technique presented in this paper is to separate the spectrums in the Fourier domain with a constant value. There are three parameters in the setup that affect the ability of the system to separate the spectrums: the tilting angle of the cube beam splitter, the angel and location of the symmetric specimen and the shearing amount. In our lab, transparent digital shearogram are recorded by using a single-cube beam splitter in a nonconventional configuration so as to both split and combine a diverging spherical wavefront emerging from a microscope objective. The camera has $1280 \times 960$ pixels, and for our experiment, we used an $11.8 \mathrm{~mm} \times 9.4-\mathrm{mm}$ camera sensor. The setup with the specific cube beam splitter produced a lateral shear of $4 \mathrm{um}$. By putting the lens array along the shearing direction, and adjusting a small shearing in $\mathrm{X}$ direction, using the method demonstrated before we can get the following result shows Fig. 3.

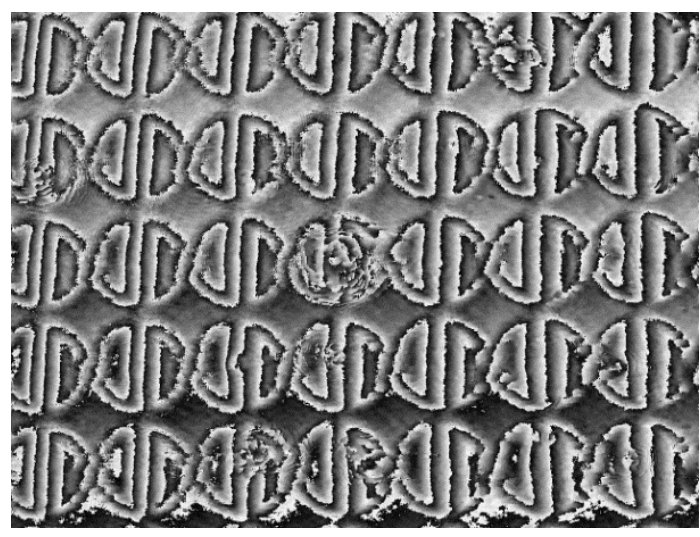

Fig. 3. Testing result of lens array. 
With different shearing amount, fringe of different type can be achieved. In this case, regarding the observation and illumination directions close to collinear, the relative phase due to shearing can then be calculated as the derivative of the testing objects. The spatial frequency carrier in this setup totally depended on the tilting angle. Once a constant spatial frequently carrier is achieved, the spectrum in Fourier domain is fixed. It will make the system more stable and robust to the vibration.

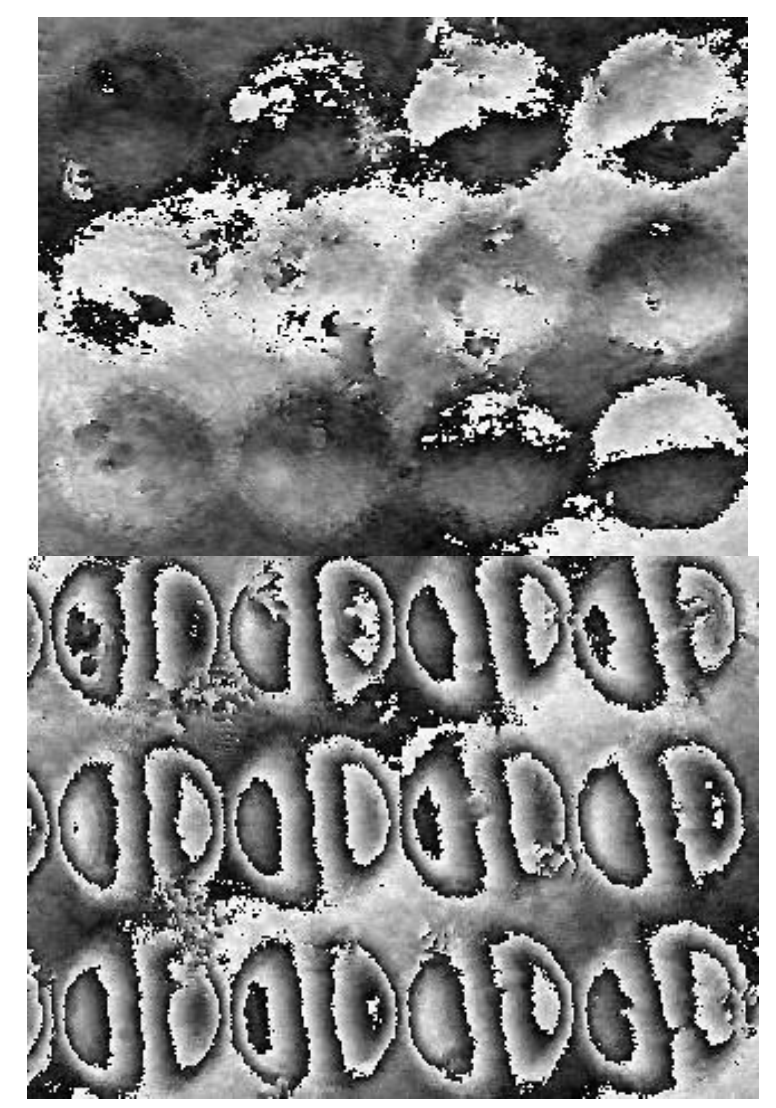

Fig.4. Comparison at different shearing sensitive at dynamic mode.

As it is a self-interference system, the angel and location of the symmetric specimen will definitely effects the result. To demonstrate that the interferometer can be used for dynamic analysis, we introduced changes in the wavefront by adjusting the location of the specimen to be tested. The location of the specimen changes the lateral shearing causing phase sensitivity changes in the collimated beam. Fig. 4 shows an image captured with the CCD at different sensitive in dynamic mode. The special frequency is the same in these different shearing conditions. 


\section{Conclusion}

In conclusion, we have demonstrated a simple yet powerful method of lateral shear interferometer. The interferometer requires just one single cube beam splitter. The advantage of using the beam splitter is that the common path beams can be made to remove it is numerical compensation in the construction process. In addition to that, in case of the single cube beam splitter, the shear is variable if the lenses are recorded on different plates. The shearing amount can be easily adjusted by moving the specimen to be tested. We have shown that it is in fact useful to have the tilt fringes because we use these fringes as carrier for the wavefront information. By taking one snap shot of the wavefront, we perform spatial Fourier transform on the recorded frame and filter one of the side bands of the spectrum using a band pass filter. The filtered spectrum is then inverse Fourier transformed to obtain the phase value of the wavefront. The experimental result with dynamic changes in the wavefront shows that this method can be used for instantaneous measurement. Also this interferometer is stable and the shearing amount can be changed without changing the special frequency carrier in harsh environment which was not possible until now.

\section{Acknowledgement}

Appreciation to d'Optron Pte Ltd Company for providing instruments, assistance with the experiments and valuable discussion.

\section{Reference}

[1] L. Xu, X. Peng, A. K. Asundi, and J. Miao, "Hybrid holographic microscope for interferometric measurement of microstructures," Opt. Eng., pp. 2533-2539, 2001.

[2] L. Xu, X. Peng, J. Miao, And, and A. K. Asundi, "Studies of digital microscopic holography with applications to microstructure testing," Appl. Opt., pp. 5046-5051.

[3] R. Groves, S. James, and RP Tatam, "Shape and slope measurement by source displacement in shearography Opt. Laser.” pp. 621-634, 2004.

[4] A. Anand, R. Groves, X. Schwab, G. Pedrini, and W. Osten, "Fresnel wavefront propagation model for shearography shape measurement," in Proc. SPIE 6617 66170Q, 2007.

[5] Joenathan C. et al, "Novel and simple lateral shear interferometer with holographic lens and spatial Fourier transform," in Opt. Eng, 2012.

[6] Joenathan C. et al, "Radial shear interferometer with holographic lenses coupled with a spatial Fourier transform method suitable for static and dynamic measurements." 2013.

[7] Q. Weijuan, C. O. Choo, V. R. Singh, Y. Yingjie, and Anand Asundi, "Quasi-physical phase compensation in digital holographic microscopy." 2009.

[8] W. Qu, Y. Yingjie, C. O. Choo, And, and Anand Asundi, "Digital holographic microscopy with physical phase compensation," in OPTICS LETTERS, 2009.

[9] A. A, G. RM, S. X, G. Pedrini, and W. Osten, "Fresnel wavefront propagation model for shearography shape measurement," in Proc. SPIE 6617 66170Q, 2007. 